\title{
Research in the Time of Coronavirus: Continuing Ongoing Studies in the Midst of the COVID-19 Pandemic
}

\author{
Parya Saberi ${ }^{1}$ \\ Published online: 18 April 2020 \\ ○) Springer Science+Business Media, LLC, part of Springer Nature 2020
}

A novel human coronavirus called severe acute respiratory syndrome coronavirus 2 (SARS-CoV-2) was identified in late 2019 in Wuhan, China, resulting in a pandemic [1]. Due to the need for physical distancing and isolation, universities closed and most medical clinics were ordered to continue with "essential" in-person visits only. In addition to a wave of medical appointment cancellations, many medical research activities, such as participant recruitment, study visits, and outcomes assessments were halted. In light of rapid societal change in response to the pandemic, this paper summarizes our considerations for conducting remote research. The discussion provided here is not exhaustive, but rather a starting place for a fundamental shift in how research is conducted.

\section{Advertisement and Recruitment}

Even without a pandemic, over $19 \%$ of trials close without meeting target accrual rates [2], underscoring the need to investigate new methods for research recruitment. Reports from the Pew Research Center show 95\%, 90\%, and $82 \%$ of individuals ages $13-17,18-29$ years, and 30-49, respectively, use some form of social media [3, 4]. This indicates a wide reach for online advertisement and recruitment for research, with the added potential to recruit populations typically considered hard-to-reach [5].

Compared to traditional recruitment methods (e.g. print and television), studies using advertisements via social media have been shown to be financially feasible, attract large numbers of individuals, and have condensed recruitment periods. They have also been shown to provide

Parya Saberi

Parya.saberi@ucsf.edu

1 Center for AIDS Prevention Studies, University of California San Francisco, UCSF, Box 0886, San Francisco, CA 94143, USA opportunities for connecting with individuals with specific health conditions, living in remote geographic locations, or who may have been difficult to recruit due to stigma or medical mistrust [6, 7]. Some examples of social media to advertise and recruit participants include: social networks (such as Facebook, Instagram, and Twitter), dating apps (Grindr, Scruff, and Jack'd), and online listservs for various medical societies or health conditions. The main disadvantage of using social media for study recruitment is the lack of reach to those with limited or no internet access. This can result in reduced generalizability of research findings [7, 8]. Researchers concerned about generalizability, however, can supplement online advertisement with traditional methods.

Another remote recruitment method involves networkbased online referral strategies. One example is respondentdriven sampling for online research which involves recruiting initial participants (i.e. "seeds") who are then asked to recruit friends to enroll in the study, and so on [9]. Finally, a host of specialist 0 medical research recruiting agencies now exist that tout increased recruitment efficiency.

\section{Screening and Enrollment}

Screening (or pre-screening) can be conducted via simple web forms on a study website. This can increase web traffic to the study website, decrease the workload for research staff, and increase a potential participant's trust in the legitimacy of the study (e.g. if the webpage is part of a reputable university with an "edu" domain). The main disadvantage of online screening is that it may either deter some to participate (e.g. if the form is too complicated or lengthy) or increase inappropriate participation (e.g. an individual or bot responding repeatedly to be eventually deemed eligible). One option to minimize these barriers is to include a simple pre-screening form on the study website and to contact the interested individual by phone call or text message to complete the screening process. 
To ensure certain key study inclusion criteria, participants can be asked to text message photographs of documents. For example, an identification card bearing their name and date of birth to verify age or a photograph of a letter of HIV diagnosis or antiretroviral medication vial bearing their name to verify HIV serostatus $[10,11]$. Signing consent and medical release forms can also be conducted online using various tools such as DocuSign or a Qualtrics survey $[10,11]$. Detailed review of the consent process is critical to ensure an individual's understanding of the study.

\section{Intervention Provision}

There are numerous ways of providing remote interventions. Text messaging has been used frequently for motivational messages, reminders, and ecological momentary assessments [12-14]. Many text messaging platforms have been developed and evaluated for HIPAA-compliant communication with participants [15].

Telehealth via videochat platforms have also been used for the provision of study interventions [16]. This modality lends itself well to interventions that do not require physical contact but are enhanced by face-to-face communication, such as interventions for mental health, smoking cessation, and medication adherence counseling. Participants in prior studies noted high acceptability levels in using videochat for research intervention delivery [17]. Benefits included being able to speak more candidly and being less intimidated, and experiencing reduced barriers for research participation (e.g. financial barriers related to travel expenses or time off from work, stigma associated with research participation, and physical disabilities precluding mobility) $[17,18]$. In addition to providing interventions, these platforms can be used for one-on-one qualitative interviews and focus groups. They allow for video- or audio-recording of the session (with consent from the participant) which can be used later for interview transcription and analysis. Other remote methods of delivering an intervention include mobile health applications [11], computers-based programs [19], and the internet [20].

\section{Monitoring and Assessment}

In addition to self-reported outcomes, which may be subject to recall and social desirability biases [21, 22], objective monitoring of study outcomes can be conducted remotely in numerous ways. For example medication adherence can be evaluated by drug hair concentrations using mail-in hair samples [23-25] and text messaged photographs of pill counts or refill dates [23-25]. Adherence has been monitored using medication event monitoring system (MEMS) caps, ingestion sensors, and "wise"-products [26-30].
Some labs provide infectious disease testing services which can examine 4th generation HIV antibody testing using dried blood spot and chlamydia and gonorrhea testing using mail-in swabs, as well as mail-in samples for Hepatitis $\mathrm{B}$ and $\mathrm{C}$ testing [31-33]. Alcohol use can be remotely monitored using Bluetooth-enabled breathalyzers that estimate breath alcohol concentration or wrist-worn alcohol biosensors that continuously measure transdermal alcohol content [34-36].

Study assessments can be conducted via online surveys which can be emailed or text messaged to participants. When surveys are conducted online, research has shown less social desirability bias, especially related to sensitive health information [37]. In cases of limited literacy, research staff can read questions to study participants or use survey platforms that include the ability to audio-record questions. Qualitative research can also be conducted using telephone, videochat, or remote focus group platforms currently typically used for marketing.

\section{Participant Incentives}

Study incentives can be provided remotely in numerous ways. For cross-sectional surveys or one-time assessments, provision of e-gift cards is the most convenient remote incentive. For longitudinal research, reloadable debit cards are a convenient method of payment and have been shown to be highly feasible and acceptable by research participants [24]. These debit cards can be mailed to participants without any funds loaded until confirmation of receipt and funds can be transferred from one card to another in case of lost or stolen cards. These are important benefits of reloadable debit cards over mailed gift cards.

\section{Conclusion}

In the era of pandemics, such SARS-CoV-2, there is a need to continue research activities, while keeping research participants and staff safe. Aligning research activities with remotely-conducted research methodology has the potential benefits of reducing time and cost for conducting the study, improving ease of participation for many individuals, enhancing the generalizability of findings, and increasing the speed of publication of study findings, all while preventing potential viral transmissions to research participants or staff.

Acknowledgements Special thanks Mr. Nikolai Caswell and Ms. Albaloo Saberi for their help on this paper. Research reported in this publication was supported by the California HIV/AIDS Research Program (CHRP) award number HD15-SF-060 (Saberi) and the National Institutes of Health award numbers R34MH114604 (Saberi) and R21MH108414 (Saberi). 


\section{References}

1. World Health Organization. Coronavirus disease (COVID-2019) situation reports. Geneva 2020 [Available from: https://www.who. int/emergencies/diseases/novel-coronavirus-2019/situation-repor ts/.

2. Kiefe CI, Sales A. A state-of-the-art conference on implementing evidence in health care-Reasons and recommendations. J Gen Intern Med. 2006;21:S67-S70.

3. Anderson M, Jingjing J. Teens, Social Media and Technology. Washington, DC: Pew Research Center; 2018 [Available from: https://www.pewresearch.org/internet/2018/05/31/teens-socia 1-media-technology-2018/.

4. Social Media Fact Sheet: Pew Research Center 2019 [Available from: https://www.pewresearch.org/internet/fact-sheet/socia 1-media/.

5. Allison S, Bauermeister JA, Bull S, Lightfoot M, Mustanski B, Shegog R, et al. The intersection of youth, technology, and new media with sexual health: moving the research agenda forward. J Adolescent Health. 2012;51(3):207-12.

6. Yuan P, Bare MG, Johnson MO, Saberi P. Using online social media for recruitment of human immunodeficiency virus-positive participants: a cross-sectional survey. J Med Internet Res. 2014;16(5):e117.

7. Whitaker C, Stevelink S, Fear N. The use of facebook in recruiting participants for health research purposes a systematic review. Journal Med Int Res. 2017;19(8): e290

8. Heiervang E, Goodman R. Advantages and limitations of webbased surveys: evidence from a child mental health survey. Soc Psych Psych Epid. 2011;46(1):69-766.

9. Wejnert C, Heckathorn DD. Web-based network sampling - Efficiency and efficacy of respondent-driven sampling for online research. Sociol Method Res. 2008;37(1):105-34.

10. Wootton AR, Legnitto DA, Gruber VA, Dawson-Rose C, Neilands $\mathrm{TB}$, Johnson MO, et al. Telehealth and texting intervention to improve HIV care engagement, mental health and substance use outcomes in youth living with HIV: a pilot feasibility and acceptability study protocol. BMJ open. 2019;9(7):e028522.

11. Erguera XA, Johnson MO, Neilands TB, Ruel T, Berrean B, Thomas S, et al. WYZ: a pilot study protocol for designing and developing a mobile health application for engagement in HIV care and medication adherence in youth and young adults living with HIV. BMJ open. 2019;9(5):e030473.

12. Mayer JE, Fontelo P. Meta-analysis on the effect of text message reminders for HIV-related compliance. Aids Care. 2017;29(4):409-17.

13. Turner CM, Arayasirikul S, Trujillo D, Le V, Wilson EC. Social inequity and structural barriers to completion of ecological momentary assessments for young men who have sex with men and trans women living with HIV in San Francisco. Jmir Mhealth Uhealth. 2019;7(5):e13241.

14. Horvath T, Azman H, Kennedy GE, Rutherford GW. Mobile phone text messaging for promoting adherence to antiretroviral therapy in patients with HIV infection. Cochrane Db Syst Rev. 2012(3).

15. Iribarren SJ, Brown W, Giguere R, Stone P, Schnall R, Staggers $\mathrm{N}$, et al. Scoping review and evaluation of SMS/text messaging platforms for mHealth projects or clinical interventions. Int J Med Inform. 2017;101:28-40.

16. Wootton AR, McCuistian C, Packard DAL, Gruber VA, Saberi P. Overcoming Technological Challenges: Lessons Learned from a Telehealth Counseling Study. Telemed E-Health. 2019.

17. Saberi P, Yuan P, John M, Sheon N, Johnson MO. A Pilot Study to Engage and Counsel HIV-Positive African American Youth Via Telehealth Technology. Aids Patient Care St. 2013;27(9):529-32.
18. Saberi P, Rose CD, Wootton AR, Ming K, Legnitto D, Jeske M, et al. Use of technology for delivery of mental health and substance use services to youth living with HIV: a mixed-methods perspective. Aids Care. 2019.

19. Kurth AE, Spielberg F, Cleland CM, Lambdin B, Bangsberg DR, Frick PA, et al. Computerized Counseling Reduces HIV-1 Viral Load and Sexual Transmission Risk: Findings From a Randomized Controlled Trial. Jaids-J Acq Imm Def. 2014;65(5):611-20.

20. Hersch RK, Cook RF, Billings DW, Kaplan S, Murray D, Safren $\mathrm{S}$, et al. Test of a Web-Based Program to Improve Adherence to HIV Medications. Aids Behav. 2013;17(9):2963-76.

21. Berg KM, Arnsten JH. Practical and conceptual challenges in measuring antiretroviral adherence. Jaids-J Acq Imm Def. 2006;43:S79-S87.

22. Kagee A, Nel A. Assessing the association between self-report items for HIV pill adherence and biological measures. Aids Care. 2012;24(11):1448-522.

23. Saberi P, Chakravarty D, Ming K, Legnitto D, Gandhi M, Johnson MO, et al. Moving Antiretroviral Adherence Assessments to the Modern Era: Correlations Among Three Novel Measures of Adherence. Aids Behav. 2019.

24. Saberi P, Ming K, Legnitto D, Neilands TB, Gandhi M. Johnson MO. Feasibility and acceptability of novel methods to estimate antiretroviral adherence: A longitudinal study. Plos One; 2019. p. 14.

25. Saberi P, Ming K, Legnitto D, Neilands TB, Gandhi M, Johnson MO. Novel methods to estimate antiretroviral adherence: protocol for a longitudinal study. Patient Prefer Adher. 2018;12:1033-42.

26. Daar ES, Rosen MI, Wang Y, Siqueiros L, Shen J, Guerrero M, et al. Real-Time and Wireless Assessment of Adherence to Antiretroviral Therapy With Co-Encapsulated Ingestion Sensor in HIV-Infected Patients: A Pilot Study. Cts-Clin Transl Sci. 2020;13(1):189-94.

27. Safren SA, Bedoya CA, O'Cleirigh C, Biello KB, Pinkston MM, Stein MD, et al. Cognitive behavioural therapy for adherence and depression in patients with HIV: a three-arm randomised controlled trial. Lancet Hiv. 2016;3(11):E529-E538538.

28. Stalter RM, Moench TR, MacQueen KM, Tolley EE, Owen DH. Consortium for ring a biomarkers and biometric measures of adherence to use of ARV-based vaginal rings. J Int AIDS Soc. 2016;19(1):20746.

29. van der Straten A, Montgomery E, Pillay D, Cheng H, Naidoo A, Cele Z, et al. Feasibility, performance, and acceptability of the wisebag (TM) for potential monitoring of daily gel applicator use in durban. South Africa Aids Behav. 2013;17(2):640-8.

30. Haberer JE, Kahane J, Kigozi I, Emenyonu N, Hunt P, Martin J, et al. Real-Time Adherence Monitoring for HIV Antiretroviral Therapy. Aids Behav. 2010;14(6):1340-6.

31. Lydie N, Saboni L, Gautier A, Brouard C, Chevaliez S, Barin F, et al. Innovative Approach for Enhancing Testing of HIV, Hepatitis B, and Hepatitis C in the General Population: Protocol for an Acceptability and Feasibility Study (BaroTest 2016). JMIR Research Protocols. 2018;7(10):109-18.

32. De Baetselier I, Smet H, Abdellati S, De Deken B, Cuylaerts V, Reyniers T, et al. (2019) Evaluation of the 'Colli-Pee', a first-void urine collection device for self-sampling at home for the detection of sexually transmitted infections, versus a routine clinic-based urine collection in a one-to-one comparison study design: efficacy and acceptability among MSM in Belgium. BMJ open. 9

33. Sharma A, Stephenson R, Sallabank G, Merrill L, Sullivan S, Gandhi M. Acceptability and feasibility of self-collecting biological specimens for hiv, sexually transmitted infection, and adherence testing among high-risk populations (project caboodle!): protocol for an exploratory mixed-methods study. JMIR Res Protocols. 2019;8(5):172-87. 
34. Fairbairn CE, Kang D. Temporal dynamics of transdermal alcohol concentration measured via new-generation wrist-worn biosensor. Alcohol Clin Exp Res. 2019;43(10):2060-9.

35. Lauckner C, Taylor E, Patel D, Whitmire A (2019) The feasibility of using smartphones and mobile breathalyzers to monitor alcohol consumpti on among people living with HIV/AIDS. Addiction science \& clinical practice, 14(1).

36. Wang Y, Fridberg DJ, Leeman RF, Cook RL, Porges EC. Wristworn alcohol biosensors: Strengths, limitations, and future directions. Alcohol. 2019;81:83-92.
37. Jones MK, Calzavara L, Allman D, Worthington CA, Tyndall M, Iveniuk J. A Comparison of Web and Telephone Responses From a National HIV and AIDS Survey. JMIR Public Health Surveill. 2016;2(2):e37.

Publisher's Note Springer Nature remains neutral with regard to jurisdictional claims in published maps and institutional affiliations. 